\title{
Laboreal
}

Volume 15 N$^{\circ} 2$ | 2019

Varia

\section{Debate de la práctica, un instrumento para la construcción de la carrera del ergónomo que traspasa las fronteras}

Debate sobre a prática, um instrumento para a construção da profissão do ergonomista que atravessa fronteiras

L'échange sur la pratique, un instrument pour la construction du métier d'ergonome qui dépasse les frontières intervention ergonomique, métier d'ergonome, réflexion sur la pratique Discussion on the practice, an instrument for the construction of the profession of ergonomist who goes beyond the borders

\section{Mirtha Mestanza y Damien Cromer}

\section{(2) OpenEdition}

Journals

\section{Edición electrónica}

URL: http://journals.openedition.org/laboreal/15497

DOI: $10.4000 /$ laboreal.15497

ISSN: 1646-5237

\section{Editor}

Universidade do Porto

\section{Referencia electrónica}

Mirtha Mestanza y Damien Cromer, « Debate de la práctica, un instrumento para la construcción de la carrera del ergónomo que traspasa las fronteras », Laboreal [En línea], Volume 15 N² | 2019,

Publicado el 01 diciembre 2019, consultado el 15 abril 2020. URL : http://journals.openedition.org/ laboreal/15497 ; DOI : https://doi.org/10.4000/laboreal.15497

Este documento fue generado automáticamente el 15 abril 2020. 


\title{
Debate de la práctica, un
} instrumento para la construcción de la carrera del ergónomo que traspasa las fronteras

\author{
Debate sobre a prática, um instrumento para a construção da profissão do \\ ergonomista que atravessa fronteiras \\ L'échange sur la pratique, un instrument pour la construction du métier \\ d'ergonome qui dépasse les frontières \\ intervention ergonomique, métier d'ergonome, réflexion sur la pratique \\ Discussion on the practice, an instrument for the construction of the profession \\ of ergonomist who goes beyond the borders
}

\section{Mirtha Mestanza y Damien Cromer}

\section{NOTA DEL EDITOR}

Manuscrito recibido en julio/2019

Aceptado tras peritaje octubre/2019

Los autores tienen a bien agradecer al conjunto de participantes a los eventos de la Comisión Jeunes Pratiques en Réflexion en Francia, Quebec y Perú. Su perennidad está enteramente ligada a su compromiso desde su creación

También, los autores tienen a bien recalcar que sin el sostenimiento continúo de la SELF y del equipo de consejo de administración y oficinas sucesivas desde el 2014 en esta aventura Finalmente, los autores desean acentuar que una Comisión funciona por el compromiso de personas las cuales están compuestas por : Sarah Couillaud, Damien Cromer, Laurène Elwert, Karen Hubert (1 $1^{\text {ra }}$ oficina Francia, 2014 - 2017) ; Patricia Bélanger, Natja Boulianne, Martin Chadoin, Céline Delga, Simon Fournier (oficina Quebec, 2014 - 2017) ; Mélissa Bohorquez, Mirtha 
Mestanza, Carolina Ullien (oficina Perú, 2018 - en curso) ; Camille Bachellerie, Manon Cléret, Damien Cromer, Julie Marié, Camille Toulisse (2da oficina Francia, 2017 - en curso).

\section{Introduccion}

2 La Comisión "Jeunes Pratiques en Réflexion" (JPR) es una emanación de la Sociedad de Ergonomía de Lengua Francesa (SELF) creada en 2014 a la iniciativa de jóvenes ergónomos practicantes de Francia y de Quebec. Esta comisión nace a partir de la constatación que los jóvenes practicantes hacen frente a dificultades particulares (Viau-Guay, 2009) y que, en la ausencia de espacios de debate de estas dificultades, éstas no hacen más que ampliarse. Para responder a esta necesidad, la Comisión ha podido realizar hasta el día de hoy 12 jornadas de debate de la práctica. Cada una de ellas, articuladas alrededor de una temática específica, ha podido reunir entre 10 y 20 ergónomos procedentes de formas diferentes de ejercicio de la carrera. Desde su creación, la Comisión se desarrolló a nivel internacional con la creación de un despacho en Quebec. Gracias a esta experiencia y con la iniciativa de jóvenes ergónomos practicantes que realizaron sus estudios de ergonomía en Francia, la Comisión ahora está presente en el Perú, desde 2018. A pesar de los países y sus diferentes contextos, los objetivos de cada despacho de la Comisión "Jeunes Pratiques en Réflexion" son los mismos : donar un espacio propicio a los debates y discusiones para favorecer el debate entre jóvenes practicantes y participar a la construcción de nuevas prácticas, permitiendo a los participantes desarrollar sus redes de contacto. Este articulo nos permitirá presentar el desarrollo de la Comisión y los aportes de esta en la práctica de los ergónomos recientemente graduados. A partir de lo que se hace en Francia y en Quebec, presentaremos los objetivos de su desarrollo recientemente en el continente sudamericano, en Perú.

\section{Del hecho a la necesidad}

Los ergónomos viven dificultades profesionales que tienen un impacto a la vez sobre ellos y sobre sus intervenciones (Viau-Guay, 2009). Como todo trabajador, el ergónomo juega su identidad, moviliza su objetividad, regula aspectos no anticipables de la intervención (Daniellou, 2006) en una perspectiva donde en pone en obra una actividad situada que implica una adaptación continua de la acción en función del contexto y que no resume solamente la aplicación de planes de acción "todos hechos" (Schön, 1987).

4 Esto es verdad a lo largo de su carrera, pero de forma particular después de sus primeros años de ejercicio (Vézina \& Baril, 2009) cuando el dispositivo de seguimiento, después de una formación inicial, no está implementado. Entonces, es en el momento de la práctica profesional que todo está flaqueando y donde los ergónomos recientemente diplomados están necesitando beneficiarse de la experiencia "de otros", para manejar las situaciones en las cuales ellos están, han estado o estarán confrontados (Beaujouan \& Daniellou 2012; Beaujouan, Coutarel \& Daniellou, 2013; Beaujouan, Aubert \& Coutarel, 2015). Los ergónomos principiantes están a la espera de la impartición de prácticas subrayando la importancia del desarrollo profesional de conocimientos y de competencias a lo largo de su recorrido profesional. Este desarrollo 
es crucial para el ergónomo que debe hacer frente a las dificultades inherentes a su práctica (Viau-Gay, 2009) : gestión de discrepancias entre los modelos de intervención e implementación real del modelo en función de los contextos : dimensión relacional de las intervenciones en ergonomía; necesidad de colaboración con ciertos actores de la empresa ; demanda de intervención yendo al encuentro de los valores del interviniente.

Es en una postura de "agente reflexivo" (Schön, 1983) que el ergónomo va poder enriquecer sus maneras de hacer y de ver sobre la base de su práctica y el de los otros. Como lo subraya Clot (2010) es en la puesta en discusión de las controversias profesionales entre profesionales que desarrollan, ellos, su práctica y por el sentido de duda, la carrera y la disciplina. Un desarrollo un tanto más indispensable a la vista de las evoluciones del mundo del trabajo y del campo de acción de la práctica de los ergónomos.

Si cada nuevo practicante viene a buscar algo específico después que participa a una jornada organizada por la Comisión "Jeunes Pratiques en Réflexion" (JPR), quisiéramos especificar su razón de ser en la continuidad de nuestro propósito y a partir de palabras que componen su denominación (Cromer, Elwert, Hubert, Francou, \& Couillaud, 2016) :

- "Jeunes" [Joven] aquí, lejos de ser tomados en flagrante delito de juvenilismo. En efecto, este calificativo se atribuye únicamente a la antigüedad en la práctica y no a la edad de los practicantes. La creación de esta comisión se basa sobre un hecho : los jóvenes practicantes tienen dificultades particulares en diferentes tipos de ejercicio de su profesión. $Y$ la ausencia del espacio de debate y de instrucción de sus dificultades no hacen más que acentuarlas. La puerta abierta dejada a los jóvenes ergónomos en su práctica es de hecho un común denominador permitiendo la creación de un colectivo facilitando el intercambio de palabras. - "Pratiques" [Prácticas]: el ergónomo es un trabajador como los otros: como todo trabajador, es en la acción que el ergónomo construye los problemas, en los cuales, él tiene que hacer frente y es la reflexión sobre la acción (Schön, 1983) que lo llevan a conocer sus propios límites y a sobrepasarlos. Las practicas están en el centro del debate y de los intercambios de palabras durante las jornadas organizadas por la Comisión. Su pluralidad es tan material permitiendo dar vida la carrera y cuestionar las reglas de la profesión para hacerla avanzar juntos (Hubault, 2003). Denominador común, ellos también, los practicantes permiten a los "estilos" (Clot, 1999) cuestionar el "tipo" común al criterio de diversos lugares del ejercicio en un contexto cambiante (Bourgeois, 2006).

- "(en) Réflexion" [Reflexionando/pensado/cuestionando]: la reflexión de las practicas demanda la construcción de un dispositivo específica permitiendo a los practicantes de traer de si durante las jornadas y de ponerlo en movimiento colectivamente. Para favorecer esta practica reflexiva colectiva, todo comienza por la explosión de una intervención cuya estructura puedo parecerse a un curso de formación (Beaujouan \& Daniellou, 2012). Lejos de ser una simple historia, esta exposición es la ocasión de pausar en una intervención que ha generado preguntas al respecto del tema propuesto. Se trata entonces, de exponer las dudas, los obstáculos, lo que se ha podido hacer y lo que no se ha podido hacer. Sigue, una discusión inicial en colectivo limitado, después en colectivo pleno permitiendo el enriquecimiento a partir de similitudes de otras intervenciones y la construcción de la reflexión común alrededor de un tema propuesto. 


\section{Contexto y nacimiento de la Comisión en Francia}

7 El censo de ergónomos efectuado por el colectivo ORME ("Organisation Représentant les Métiers de l'Ergonomie") en 2015 (Bonnin, Rascle, \& Roger, 2017) ha permitido estimar el número de ergónomos a más de dos mil en Francia. Su número esta en crecimiento hace 50 años y las formas de ejercicio de la carrera son múltiples: consultores, internos, en servicio de salud en el trabajo (SST), en investigación, etc. También, un título protegido a la escala de Europa (el título de ergónomo Europeo) permite calificar a los ergónomos de más de 3 años de ejercicio que hacen la solicitud y la formación, la trayectoria y la práctica correspondiente a ciertos criterios. Desde el 2018, certificación "ergónomo junior certificado" viene a completar una necesidad complementaria: permite a los nuevos profesionales hacer valorar la calidad de su formación inicial, ya que ellos no pueden todavía acceder al título de Ergónomo Europeo en ejercicio.

8 En este contexto de desarrollo de la carrera y de la disciplina, muchas asociaciones participan a esta estructuración. La SELF creada en 1963 es la primera de entre ellas seguida recientemente por asociaciones profesionales, locales o todavía de estudiantes. También, cada año muchos congresos o jornadas de estudios son realizadas por ciertas universidades o asociaciones dentro de ello el congreso anual de la SELF.

9 A pesar de esta estructuración, el constatado hecho en 2014 es que ha sido poco frecuente ver jóvenes practicantes aventurarse y participar a los debates después de las presentaciones durante los congresos animados por la comunidad. Muchos factores pueden explicar esta reserva y notablemente el hecho de un cierto miedo al juzgamiento. Estos temores pueden igualmente expresarse dentro de estructuras que proponen momentos de los intercambios de palabras. Este hecho ha llevado a los miembros fundadores de la Comisión a identificar una necesidad de compartir alrededor de la práctica de la carrera del ergónomo y para que este debate pueda aprovechar a los jóvenes ergónomos, era necesario que la palabra pueda ser liberada. Esta libertad parece poder tener lugar solamente entre "nosotros" los jóvenes ergónomos, que era necesario crear un "entre sí" efímero y recurrente.

10 A la escucha de este hecho y de los jóvenes practicantes que lo portan, la SELF permite la apertura de la Comisión Jeunes Pratiques en Réflexion. Su creacion dentro de la SELF hace sentido en un sentido integrativo de diálogo con las otras asociaciones ya presentes en la comunidad francófona. Era necesario todavía definir lo que "jóvenes" quiere decir. "El entre sí" necesita la reunión de individuos teniendo un nivel de experiencias similares o próximas y es para favorecer los debates y las comparaciones de prácticas entre profesionales. También, después de una reflexión madura, ha sido decidido abrir estos espacios de discusión a los participantes de menos de 6 años de experiencia en ergonomía cualquiera sea su edad. Validado en acta, la Comisión ha podido comenzar su trabajo.

\section{Construcción de la comisión en Francia}

Presentaremos aquí algunos elementos sobre la estructuración, así como también la ingeniería de la discusión implementada en Francia. La filosofía de fondo es invariable 
en sus duplicaciones en Quebec, después hoy en día en Perú. Las siguientes partes nos permiten evocar e interrogar las especificaciones locales.

12 En Francia después de su creación, la Comisión ha realizado 12 jornadas de debates sobre la práctica. Las cuales han reunido una quincena de ergónomos recientemente graduados llegando a más de 150 el número de participantes después de 5 años de actividad. Cada jornada está articulada alrededor de una temática especifica (e.g., : "la pluridisiplinariedad en la intervención"; "la promoción de la intervención ergonómica"; "el ergónomo en búsqueda de profesionalización"; "la caja a herramientas del ergónomo"). Estas temáticas son construidas a partir de un sondaje anual en línea a fin de abarcar al mayor número, respondiendo a las problemáticas concretas que preocupan la práctica cotidiana.

Las jornadas son animadas por dos animadores en el cual el rol, aparte de animar es de asegurarse de la participación activa de los participantes favoreciendo el debate. Detallaremos la organización de estas jornadas en tres tiempos : (1) antes de la jornada ; (2) la jornada ; (3) después de la jornada.

\subsection{Antes de la jornada}

14 Previamente a la jornada, una organización metodológica de ésta a la vista de los objetivos de la Comisión resulta indispensable (Clot, Faïta, Fernandez, \& Scheller, 2000). Así, el animador procede al trabajo "de ingeniería y discusión" (Detchessahar, 2011; Van Belleghem \& Forcioli-Conti, 2015). El crea las condiciones que serán necesarias a la emergencia y la realización efectiva del debate de las prácticas y la actividad real. Otro, los aspectos logísticos, el animador establece el desarrollo de la jornada vigilando cuadrarla sin atraer la rigidez, a fin de dejar lugar a los debates. El define así los diferentes tiempos de la jornada, las orientaciones dadas, y pre-identifica los momentos de regulación de la jornada. El organiza la jornada en función del panel de inscritos con el fin de favorecer el debate de las practicas entre ergónomos de estructuras heterogéneas. Aparte del contenido, el animador vigila también el contenido de la jornada. El identifica puntos alrededor de los cuales los participantes intercambiaran a fin de asegurarse que cada uno pueda expresarse y que el grupo tome una pausa sobre la temática propuesta y sobre su práctica. Los participantes se inscriben con anticipación vía un sondeo en línea y proponen una presentación voluntaria.

\subsection{La jornada}

Durante la jornada, el animador debe hacer emerger los debates de las practicas :

- El primer tiempo consiste en abrir la jornada. Cada participante se presenta y expresa sus expectativas. Las reglas de funcionamiento del grupo son construidas con el fin de instaurar un clima de escucha, de confianza y de benevolencia, permitiendo la libre expresión de cada uno y la disputa profesional. La importancia del marco propuesto, en la cual los animadores son los garantes es primordial en el transcurso de esta jornada.

- El segundo tiempo consiste en debatir colectivamente en grupos pequeños. En efecto, los sub-grupos son preparados con anticipación, una discusión situada es lanzada, permitiendo en lo consecutivo a los participantes evocar situaciones de intervenciones en conexión y de alargar la dinámica del cuestionamiento a todo el grupo. Durante esta fase, los animadores 
navegan de grupo en grupo a fin de asegurar que los debates y la "mayéutica" tengan lugar $\mathrm{y}$, como responsable del marco, intervienen si hay necesidad.

- La tercera parte consiste en una difusión de los debates que hayan tenido lugar en los subgrupos. Seguido a la fase de restitución, un debate con todos los participantes es iniciado. Los animadores, en cuanto a ellos, sintetizan los puntos más destacados de cada restitución y acompañan vinculando los diferentes elementos evocados.

\subsection{Después de la jornada} basan esencialmente en el último momento de la jornada para construir un soporte de integración y de nuevas reflexiones seguido a los debates. La idea no es de producir un documento analítico y exhaustivo, pero de "retranscribir" las preguntas acentuadas, los obstáculos y las fortalezas identificadas, poniendo en evidencia las conexiones que pudieron ser hechas. Este documento es únicamente a destinación de los participantes de la jornada, permite acceder a un nivel superior de "sedimentación" de los debates.

El análisis de estos cinco años de funcionamiento nos ha permitido hacer evolucionar las formas de debates y de convocatoria de la actividad de los participantes. También, cada temática llama a una modificación específica guardando los elementos de estructuración invariable.

Las opiniones de los participantes son numerosas. Muy a menudo en caliente, pero también después y son a distintos niveles. De una parte, los participantes ponen en evidencia la importancia de la creación de redes; no es de hecho raro que los participantes se llamen o llamen a los miembros de la Comisión fuera de las jornadas. Por otra parte, a nivel individual los elementos de debate han permitido resolver situaciones concretas en el ejercicio de los participantes. A nivel colectivo, ciertos participantes han podido trasponer este formato de debates en la práctica con sus colegas dentro de su estructura de pertenencia. Después de un Symposium (Cromer et al., 2016), dejamos el lugar a un participante. Este ha podido evocar de forma espontánea "en su práctica profesional, los debates de la jornada lo han ayudado, en cierta medida, a evolucionar su manera de comprender las intervenciones". También, concluyó su intervención diciendo : "mi participación en esta jornada a dado un nuevo ímpetu y una nueva dinámica al ejercicio de mi carrera de intervencionista. La Comisión representa un espacio privilegiado, un apoyo para "reinterrogar" y hacer evolucionar nuestras prácticas sobre la base de retornos de experiencia".

\section{Una primera experiencia tras-Atlántica : Quebec}

A partir de su construcción en Francia, la Comisión ha encontrado muy rápido un eco a cargo de jóvenes practicantes en el extranjero y en ocurrencia en Quebec. Desarrollaremos en seguida la apropiación peruana actual, deseamos evocar la experiencia desarrollada en Quebec de 2014 a 2017 y hoy en día pendiente, esperando nuevos voluntarios para volver a implantarlo. En Quebec, el número de ergónomos se sitúa entre 250 y 300 (Lortie, 2009) por tres formaciones en ergonomía impartidas en la universidad. Dos asociaciones estructuran el pasaje: uno a la escala de Canadá ("l'Association Canadienne d'Ergonomie" - ACE) y el otro más reciente en Quebec ("l'Association Professionnelle des Ergonomes du Québec" - APEQ). Sobre una 
superficie de más de 1,5 millones de $\mathrm{km}^{2}$, los ergónomos resultan dispersos en el territorio lo que no facilita las posibilidades de reagrupamiento y de debates entre practicantes. La mayoría de entre ellos trabajan en demandas orientadas por problemáticas de salud (indemnización, readaptación, TMS, ergonomía de oficina). También, el hecho de su proximidad con los Estados Unidos hace que esté presente una influencia de la ergonomía de Factores Humanos.

En este contexto, en Francia pudimos desde el principio favorecer el debate sobre la práctica, la función de la Comisión en Quebec era también de contribuir a la estructuración de una comunidad pluralista (Chadoin, Boulianne, Hubert, \& Couillaud, 2016). Así, el objetivo ha sido de llegar a consolidar una comunidad a través de los debates entre jóvenes practicantes para identificar los puntos comunes que hacen "comunidad", pero también para romper el aislamiento profesional. Las empresas de Quebec no permiten la realización de estas jornadas dentro del tiempo de trabajo de los ergónomos, los eventos organizados en Quebec se desarrollaban al finalizar el día. Como para Francia, los eventos se articulaban alrededor de temáticas (e.g., "el trabajo del ergónomo en lo cotidiano, posicionamiento, colaboraciones, desafíos, etc.”).

Estos eventos agrupaban una decena de jóvenes practicantes articulándose, como en Francia, en diferentes tiempos. En un inicio, dos o tres ergónomos han sido invitados a atestiguar sus prácticas en función de un sujeto. Ellos disponían de un tiempo para presentarse y presentar su estructura, pues su exposición de intervención en conexión con la temática. Durante este tiempo, toda interrupción era posible a fin de anclar formas de comparaciones de prácticas entre los participantes.

Estos eventos han podido desarrollarse durante los tres años de funcionamiento del despacho en Quebec permitiendo el intercambio de prácticas difíciles tanto del punto de vista de formaciones iniciales de los participantes (ergonomía de la actividad / Human Factors) que solo del punto de vista de alejamiento territorial.

\section{La comisión en Perú}

No se encontró una traducción literal del nombre de la Comisión a español, pero las JPR son tituladas : "Jornadas de Jóvenes Ergónomos en Perú".

Los países de América del sur al igual que Quebec se ven muy influenciados por las dos corrientes de ergonomía : Ergonomía de la actividad / Human Factors. En este contexto en el Perú encontramos profesionales con formación pura en una de las dos corrientes de ergonomía o una formación mixta ya que actualmente, sólo hay dos maestrías en dos universidades de Lima. Estos profesionales se desempeñan en diversos sectores como: minería, industria, agricultura, salud, etc.

Hay una gran cantidad de médicos del trabajo, ingenieros, entre otros profesionales, que tienen la formación de ergonomía y utilizan sus conocimientos para la gestión de la salud ocupacional en las empresas, consultores externos o profesores en las universidades.

Los jóvenes practicantes se ven envueltos en las dos formas de prácticas de la ergonomía. Es cierto que muchos de ellos practican la ergonomía de forma empírica porque cuentan con alguna pequeña formación (diplomado, cursos de universidad, cursos de especialización, etc.). Entonces, ellos sólo responden directamente a la demanda inicial de la empresa, generalmente realizar una medición de ángulos de la 
postura de trabajo de la persona, aplicar un método de evaluación del riesgo y recomendar algunas soluciones. Esto para el cumplimiento de las exigencias legales o normativas de la empresa. Otros, por el contrario, cuentan con maestrías en el extranjero y tratan de reformular la demanda para generar una intervención ergonómica que genere en el mejor de los casos la transformación del trabajo o en su defecto un impacto positivo en los actores de la empresa.

Las demandas en su mayoría emergen por problemas asociados a Trastornos Musculo Esqueléticos o un alto índice de días de descanso médico. Es el médico del trabajo, responsable de higiene y seguridad o el gerente de recursos humanos quien porta en su mayoría las solicitudes de intervención. En muy pocas ocasiones se desarrollan intervenciones ergonómicas, pero éstas van tomando un importante posicionamiento gracias a los resultados evidenciados en las empresas y el desarrollo de diplomados de ergonomía con un enfoque sistémico.

Gracias a la formación francesa de algunos ergónomos practicantes en Perú actualmente, conociendo la importancia de la práctica reflexiva en las formaciones en Francia e inspirados en el éxito de las jornadas de "Jeunes Pratiques en Réflexion" nos hemos visto en la necesidad de generar espacios de reflexión de la práctica de la ergonomía en Perú.

El objetivo queda invariable, es decir la generación de espacios de debate para los practicantes recién graduados, pero a raíz de las necesidades de formación, durante las jornadas se toma un tiempo para la formación técnica, recomendación bibliográfica de la ergonomía de la actividad o ergonomía sistémica y el desarrollo de una intervención ergonómica.

Hasta la fecha hemos realizado dos jornadas, una en Lima y otra en Arequipa y éstas reunieron en total 12 participantes. El contexto laboral exige generalmente realizarlo los fines de semana (viernes por la tarde, sábados o domingos).

31 Las jornadas son organizadas bajo la misma metodología de la matriz Francia, teniendo en cuenta ciertas modificaciones : tiempo para la impartición de conocimientos de la ergonomía de la actividad, la exposición de casos de accidentes de trabajo, el desarrollo de proyectos de investigación, las intervenciones bajo la ergonomía Humans Factors y por su puesto las intervenciones bajo la ergonomía de la actividad. Debido al pequeño número de participantes, hemos resuelto la exposición de cada caso frente a todos y el debate ha estado focalizado en : "la construcción de la intervención, el posicionamiento y la transformación del trabajo".

32 De la primera jornada hemos tenido la retroalimentación de algunos participantes. Uno de ellos nos dijo lo siguiente :

"la razón por la cual participé en la jornada es porque buscaba casos reales que otros participantes hayan solucionado. Esta jornada se diferenció de otras porque tuve la oportunidad de contar mis errores, fallas, éxitos y avances en la gestión. Además, me beneficie de la experiencia de los otros participantes. Me quedó grabado la importancia del posicionamiento para lograr la transformación del trabajo. A partir de esa jornada abordo los problemas en mi empresa de forma sistémica, no busco una solución inmediata, sino tomo una pausa para encontrar la causa raíz del problema. En las próximas jornadas espero encontrar experiencias de otros rubros y las diferentes formas de solucionar los problemas para de esta manera abrir mi mente a nuevas ideas". 

jóvenes practicantes puedan dialogar en un marco favoreciendo el "entre nosotros", pensamos que éste no tiene sentido que en la aplicación dinámica con la totalidad de la comunidad profesional (Cromer, Cléret, Marié, Toulisse, Chambel, \& Bachellerie, 2019). Ha sido el objetivo de este artículo : dar a conocer los objetivos de la fabricación, desde este año, talleres de dialogo sobre la practica en el Perú. Estos últimos, son el fruto de una construcción más antigua que ha comenzado y funciona hasta el día de hoy en Francia. organizar estos espacios de debate sobre la práctica, hemos podido poner en evidencia los objetivos comunes. El ergónomo defiende un enfoque de desarrollo conjunto de la eficacia y de la salud en la empresa, y es con estos dos objetivos centrales que se juega toda la necesidad del debate sobre la práctica. Frente a contextos de intervenciones múltiples y siempre complejas, el ergónomo se apoya sobre sus recursos (formación inicial, colegas, supervisor, etc.), pero las situaciones de aislamiento y/o de límite de estos recursos pueden resultar numerosos (Viau-Guay, 2009). Es aquí mismo que se sitúan los objetivos de esta Comisión : frente al hecho de diversidad y de diversificación de prácticas, ser espacio de debate y de reflexión sobre las practicas actuales creados por y para los jóvenes practicantes Lugares de debates que permiten a los practicantes desarrollar individual y colectivamente su práctica a través de relatos de lo que Ellos han podido hacer, pero también lo que no han podido hacer. Si este punto es convergente en Francia, Quebec y Perú podemos ver que las divergencias de contextos entre estos países conllevan a diferentes objetivos dentro de la emergencia y la estructuración de la comunidad de práctica. En todos los casos, estas tres experiencias desarrolladas por la Comisión ponen en evidencia la necesidad de un marco permitiendo estos desarrollos de la práctica de la carrera del ergónomo a la escala individual, colectiva e institucional. $Y$ es en esta perspectiva y en un dialogo constante entre practicantes recientemente graduados y toda la comunidad nacional e internacional de la Comisión se propone proseguir su contribución para los futuros años. 


\section{BIBLIOGRAFÍA}

Beaujouan, J., \& Daniellou, F. (2012). Les récits professionnels dans une formation d'ergonomes. Le Travail Humain, 4(75), 353-376. doi : 10.3917/th.754.0353

Beaujouan, J., Coutarel, F., \& Daniellou, F. (2013). L'expérience des autres dans la formation : apports et limites du récit professionnel. Éducation Permanente, 196, 25-38.

Beaujouan, J., Aubert, S., \& Coutarel, F. (2015). Construction de l'intervention ergonomique. D'une préoccupation de montée en cadence à la décision d'investir pour transformer le travail : embûches et stratégies. Perspectives interdisciplinaires sur le travail et la santé, 17(2). doi : 10.4000/ pistes.4599

Bonnin, D., Rascle V., \& Roger, T. (2017). Ergonomes praticiens et ergonomie opérationnelle : quels ancrages et quels moyens ? Problématiques actuelles de l'organisation du métier. In B. Barthe, O. Gonon, \& C. Brun (Coord.), Actes du $52^{\text {ème }}$ Congrès de la SELF : Présent et futur de L'Ergonomie (pp. 709-711). Toulouse : SELF.

Bourgeois, F. (2006). Déterminer la valeur et l'importance d'une intervention : l'ergonome confronté encore à la mesure, à la subjectivité et à la finalité de son action. In Collection Journées de Bordeaux sur la pratique de l'ergonomie : La place de l'évaluation dans la pratique de l'ergonomie (pp. 113-121). Université Victor Segalen Bordeaux 2.

Chadoin, M., Boulianne, N., Hubert, K., \& Couillaud, S. (2016). Un processus commun de construction des pratiques, mais des moyens et des temporalités différents

L'exemple de la Commission SELF Jeunes Pratiques en Réflexion en France et au Québec. Congrès de l'ADÉO-L'ergonomie de l'activité à travers le Monde.

Clot, Y. (1999). La fonction psychologique du travail. Paris : PUF.

Clot, Y. (2010). Le travail à cœur. Pour en finir avec les risques psychosociaux. Paris : La Découverte.

Clot, Y., Faita, D., Fernandez, G., \& Scheller, L. (2000). Entretiens en autoconfrontation croisée : une méthode en clinique de l'activité. Perspectives interdisciplinaires sur le travail et la santé, 2(1). doi : $10.4000 /$ pistes.3833

Cromer, D., Elwert, L., Hubert, K., Francou, F., \& Couillaud, S. (2016). La commission SELF Jeunes Pratiques en Réflexion : de l'échange sur la pratique à son enrichissement. In S. Leduc, \& G.

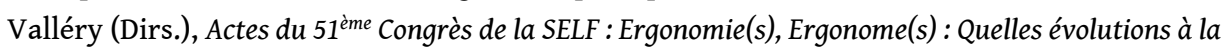
croisée d'une discipline et d'un métier? Marseille : SELF.

Cromer, D., Cléret, M., Marié, J., Toulisse, C., Chambel, A., \& Bachellerie, C. (2019). Quand les expériences se rencontrent : une opportunité de développement des pratiques de chacun Ateliers d'échanges sur les pratiques ouverts à tous. In Actes du $54^{\text {ème }}$ Congrès de la SELF - Université de l'Ergonomie: Comment contribuer à un autre monde? Tours : SELF.

Daniellou, F. (2006). Entre expérimentation réglée et expérience vécue : Les dimensions subjectives de l'activité de l'ergonome en intervention. @ctivités 3(1), 5-18. doi : 10.4000/activites. 1835

Detchessahar, M. (2001). Quand discuter, c'est produire... Pour une théorie de l'espace de discussion en situation de gestion. Revue française de gestion, 132, 32-43.

Hubault, F. (2003). Impossible de s'entendre si on renonce à danser ensemble. In F. Hubault (Coord.), Le métier d'ergonome (pp. 155-169). Toulouse : Éditions Octarès. 
Lortie, M. (2009). L'ergonomie au Québec : perspectives et prospectives. Perspectives interdisciplinaires sur le travail et la santé, 11(1). doi : 10.4000/pistes.2902

Schön, D. (1983). The reflexive practitioner : how professionals think in action. USA : Basic Books.

Schön, D. (1987). Educating the Reflective Practitioner. San Francisco : Jossey-Bass.

Van Belleghem, L., \& Forcioli Conti, E. (2015). Une ingénierie de la discussion ? Chiche! In Actes du $50^{\text {ème }}$ Congrès de la SELF : Articulation performance et santé dans l'évolution des systèmes de production (pp. 323-331). Paris : SELF.

Vézina, N., \& Baril, R. (2009). Apprendre à intervenir : difficultés rencontrées par de jeunes

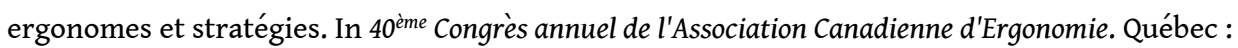
ACE.

Viau-Guay, A. (2009). Analyse de l'activité déployée par un ergonome lors de difficultés professionnelles. Contribution à la formation initiale (Thèse de doctorat). Faculté des Études Supérieures, Université Laval, Quebec.

\section{RESÚMENES}

Desde este año, espacios de debate de la práctica de la carrera creada por y para jóvenes practicantes en ergonomía son implementados en Perú. Esta iniciativa parte del hecho que el ergónomo es un trabajador como otro, y que, a este título, es necesario dialogar sobre la acción que realiza cotidianamente para avanzar y construir su práctica futura. Si estos espacios están recientemente en Perú, éstos existen en Francia hace 5 años y son propuestos por la Comisión "Jeunes Pratiques en Réflexion" de la sociedad de Ergonomía de Lengua Francesa. En este artículo, proponemos contar su génesis, su funcionamiento y los objetivos de su desarrollo.

Desde este ano, espaços de debate sobre a prática do oficio, criados por e para jovens profissionais em ergonomia, foram implementados no Peru. Essa iniciativa baseia-se no fato de que o ergonomista é um trabalhador como qualquer outro, e que, por isso, é necessário discutir a ação realizada diariamente afim de progredir na construção da sua prática futura. Se esses espaços são recentes no Peru, eles existem na França há já 5 anos e são propostos pela comissão "Jeunes Pratiques en Réflexion" da Sociedade de Ergonomia Francesa (SELF). Neste artigo, propomos contar sua gênese, seu funcionamento e os desafios dos seus desenvolvimentos.

Depuis cette année, des espaces d'échanges sur la pratique du métier créés par et pour de jeunes praticiens en ergonomie sont mis en place au Pérou. Cette initiative part du constat que l'ergonome est un opérateur comme un autre, et qu'à ce titre il a besoin d'échanger sur l'action qu'il mène quotidiennement pour avancer et construire sa pratique de demain. Si ces espaces sont récents au Pérou, ils existent depuis plus de 5 ans en France et sont proposés par la Commission “Jeune Pratiques en Réflexion» de la Société d'Ergonomie de Langue Française. Dans cet article, nous proposerons d'en raconter la genèse, le fonctionnement et les enjeux de leur développement. 


\section{ÍNDICE}

Palavras-chave: intervenção ergonômica, profissão de ergonomista, reflexão sobre a prática Keywords: This year, spaces to exchange practices have been created by and for young ergonomics practitioners in Peru. This initiative stems from the observation that the ergonomist is an operator as any another, and that as such it is necessary to discuss the daily action in order to advance and build tomorrow's practice. If these spaces are recent in Peru, they exist for more than 5 years in France and are proposed by the Committee "Jeunes Pratiques en Réflexion" from the French Speaking Ergonomics Society (SELF). In this paper, we propose to tell their genesis, their functioning and the challenges of their development. rgonomic intervention, ergonomist profession, reflection on practice

Palabras claves: intervención ergonómica, profesión ergónomo, reflexión sobre la práctica

\section{AUTORES}

\section{MIRTHA MESTANZA}

Aquaergo / Pontificia universidad católica del Perú, Av. Universitaria 1801, San Miguel, Lima 32, Perú mmestanzat@pucp.edu.pe

\section{DAMIEN CROMER}

Ousia Conseil / Laboratoire ACTé (EA 4281), Université Clermont Auvergne, 34 Avenue Carnot, 63000 Clermont-Ferrand, France

damien.cromer@uca.fr 